\title{
Rotura diafragmática no trauma abdominal fechado
}

\author{
Blunt traumatic diaphragmatic rupture
}

\begin{abstract}
Antonio Carlos Nogueira ${ }^{a}$, Munir Bazzi ${ }^{b}$, Francisco Garcia Soriano ${ }^{a}$, Haydée Jordãoc ${ }^{c}$ Sueli Vieira Lopes ${ }^{c}$, Sebastião Pontes ${ }^{c}$, Ana Carolina Makino Antunes ${ }^{d}$, João Augusto dos Santos Martines ${ }^{e}$
\end{abstract}

Nogueira AC, Bazzi M, Soriano FG, et al. Rotura diafragmática no trauma abdominal fechado. Autopsy Case Rep [Internet]. 2011;1(3):39-45. http://dx.doi.org/10.4322/acr.2011.007

\section{RESUMO}

A incidência de lesão traumática do diafragma, relatada na literatura, varia de 0,6 a $1,2 \%$ dentre os pacientes vítimas de traumas, elevando-se para $5 \%$ nos pacientes com trauma fechado submetidos a laparotomia. A suspeita clínica associada à avaliação radiológica contribui para o diagnóstico precoce. A lesão diafragmática isoladamente é de bom prognóstico. Assim, em geral, as lesões associadas à rotura diafragmática são os preditores da pior evolução do paciente. As lesões do diafragma direito e as lesões bilaterais apresentam pior prognóstico. A tomografia computadorizada com multidetectores (MDCT) de tórax oferece a possibilidade de reconstrução multiplanar permitindo melhor acurácia no diagnóstico. A correção cirúrgica por meio de laparotomia e/ou toracotomia na fase aguda do trauma apresenta boa evolução e evita as complicações crônicas da hérnia diafragmática. Os autores apresentam o caso de um paciente jovem do sexo masculino, vítima de trauma abdominal fechado por acidente automobilístico que apresentou rotura do diafragma, lesão esplênica e renal. O diagnóstico foi feito através da tomografia computadorizada de tórax e abdome e confirmada durante laparotomia exploradora.

Unitermos: Diafragma; Hérnia; Laparotomia; Ferimentos e lesões.

\section{ABSTRACT}

Traumatic injury of the diaphragm ranges from 0.6 to $1.2 \%$ and rise up to $5 \%$ among patients who were victims of blunt trauma and underwent laparotomy. Clinical suspicion associated with radiological assessment contributes to early diagnosis. Isolated diaphragmatic injury has a good prognosis. Generally worse outcomes are associated with other trauma injuries. Bilateral and right diaphragmatic lesions have worse prognosis. Multi detector computed

\footnotetext{
a Divisão de Clínica Médica do Hospital Universitário - Universidade de São Paulo, São Paulo/SP - Brasil.

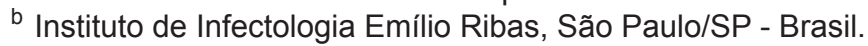

c Hospital das Clínicas da Faculdade de Medicina - Universidade de São Paulo, São Paulo/SP - Brasil.

d Divisão de Clínica Cirúrgica do Hospital Universitário - Universidade de São Paulo, São Paulo/SP - Brasil.

e Serviço de Imagenologia do Hospital Universitário - Universidade de São Paulo, São Paulo/SP - Brasil.
}

Copyright $\odot 2011$ Autopsy and Case Reports - Este artigo de Acesso Aberto é distribuído pelos termos do Creative Commons Attribution NonCommercial License (http://creativecommons.org/licenses/by/3.0/) que permite livre uso não-comercial, distribuição e reprodução em qualquer meio, desde que os artigos sejam devidamente citados. 
tomography (MDCT) scan of the chest and abdomen provides better diagnostic accuracy using the possibility of image multiplanar reconstruction. Surgical repair via laparotomy and/ or thoracotomy in the acute phase of the injury has a better outcome and avoids chronic complications of diaphragmatic hernia. The authors present the case of a young male patient, victim of blunt abdominal trauma due to motor vehicle accident with rupture of the diaphragm, spleen and kidney injuries. The diagnosis was made by computed tomography of the thorax and abdomen and was confirmed during laparotomy.

Keywords: Diaphargm; Hernia; Laparotomy; Wounds and injuries.

\section{CASO CLÍNICO}

Homem de 25 anos foi admitido no prontosocorro do Hospital Universitário da Universidade de São Paulo, vítima de colisão de motocicleta com automóvel com trauma da região costal anterior esquerda.

Durante $o$ transporte 0 paciente não apresentou redução ou alteração do nível de consciência, porém foi relatado, pelos socorristas, um episódio de êmese. Apresentava-se à admissão consciente (escala de Glasgow $=15$ ), imobilizado em prancha rígida com colar cervical, freqüência cardíaca de 75 batimentos por minuto e respiratória de 16 incursões por minuto, saturação de $\mathrm{O}_{2}$ de $100 \%$ em ar ambiente, pressão arterial de $110 \times 75 \mathrm{~mm}$ de $\mathrm{Hg}$. Queixava-se de dor nos ombros, membro superior direito e região torácica anterior à esquerda. Não apresentava achados anormais na ausculta cardiopulmonar nem alterações na avaliação neurológica. Evidenciavase pequena exteriorização de sangue em cavidade oral, sem lesões visíveis.

Submetido a exames de imagem: as radiografias da bacia, membros superiores $e$ inferiores não revelaram fraturas. A tomografia computadorizada de crânio não apresentava alterações.

As tomografias de tórax e abdome revelaram hérnia diafragmática à esquerda contendo estômago dilatado e alça de cólon, fratura esplênica, laceração renal esquerda e hemoperitônio de moderado volume (Figuras de 1 a 4 ).

Submetido a laparotomia de urgência, encontrou-se moderada quantidade de coágulos na cavidade abdominal, rotura diafragmática de aproximadamente $10 \mathrm{~cm}$ iniciando próximo ao hiato esofágico e estendendo-se lateralmente à parede do hemitórax esquerdo, com herniação do estômago para a cavidade torácica, ruptura esplênica e hematoma retroperitoneal próximo ao rim esquerdo. Realizada esplenectomia, tamponamento do hematoma retroperitoneal com gelfoan, redução do estômago para cavidade abdominal, drenagem de tórax sob visão direta e rafia da ruptura diafragmática com pontos separados de vicryl 1.

Permaneceu na UTI por 5 dias, recebeu alta para enfermaria, com boa evolução recebendo alta hospitalar após 7 dias da admissão. O controle tomográfico no $15^{\circ}$ dia de pós operatório mostrou integridade do contorno diafragmático esquerdo.

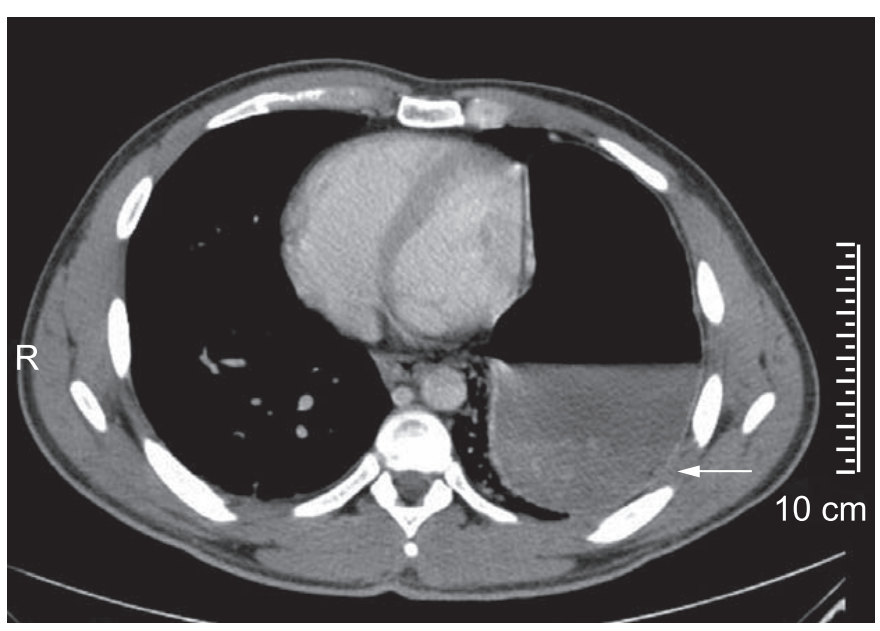

Figura 1 - Imagem MDCT tórax no plano axial mostrando estômago intratorácico dilatado tocando as costelas posteriores (sinal de víscera dependente - seta). 


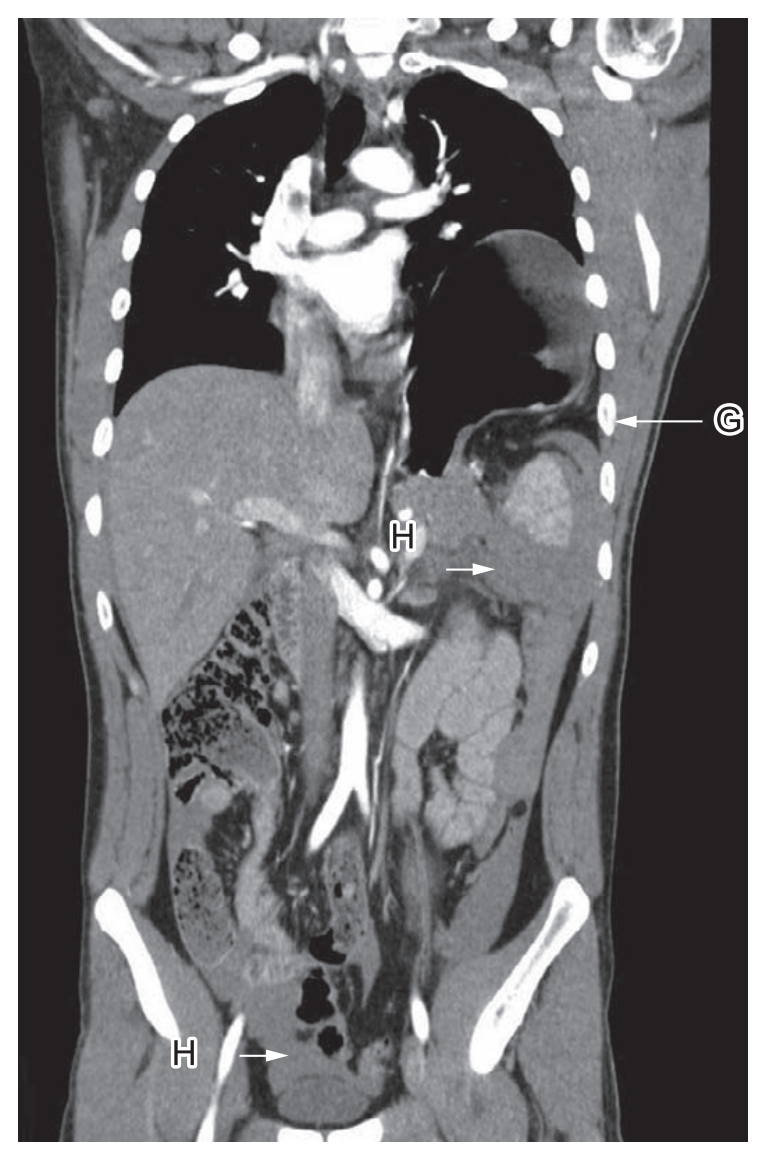

Figura 2 - Imagem MDCT tórax e abdômen, com reformatação coronal, evidenciando descontinuidade do diafragma com estômago dilatado intratorácico e herniação da gordura abdominal (seta G), hemoperitônio de moderado volume (setas $\mathrm{H}$ ).

\section{DISCUSSÃO}

A incidência exata das lesões traumáticas do diafragma é difícil de ser determinada devido ao grande número de diagnósticos não realizados na fase aguda da lesão ou feitos tardiamente, além da ocorrência de mortes pré-hospitalares. ${ }^{1}$ Entretanto sabe-se que a rotura traumática do diafragma é uma ocorrência incomum cuja incidência relatada em dois bancos de dados de trauma dos EUA é de 0,63 a $1,2 \% .^{2,3}$ Esta incidência eleva-se para $2,1 \%$ quando considerados somente os traumas tóraco-abdominais fechados ${ }^{1}$, e para $5 \%$ quando considerados os traumas tóracoabdominais operados. ${ }^{4,5}$ As lesões diafragmáticas ocorrem principalmente por colisão de veículos motorizados (80-90\%) sendo aproximadamente $75 \%$ causadas por trauma fechado ${ }^{6}$ e as vítimas são predominantemente do sexo masculino na faixa etária jovem, como observado no caso apresentado. $^{7}$

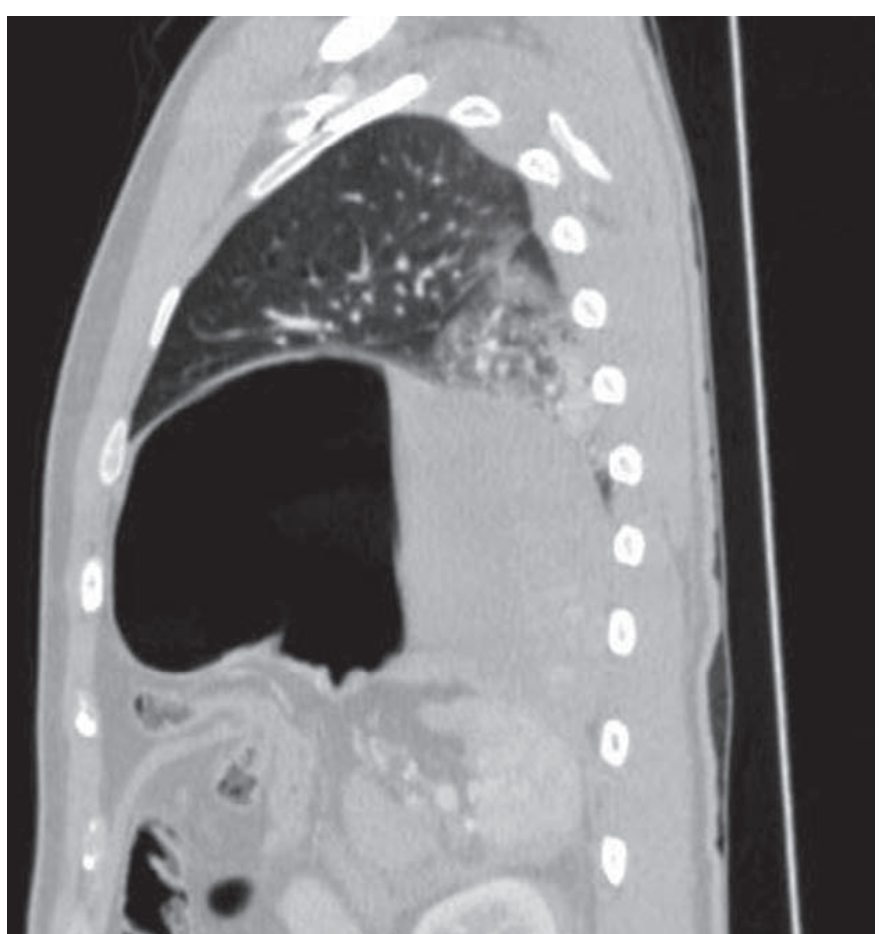

Figura 3 - Imagem MDCT tórax e abdômen, plano sagital, janela pulmonar, mostrando hérnia diafragmática e atelectasia passiva do lobo inferior esquerdo.

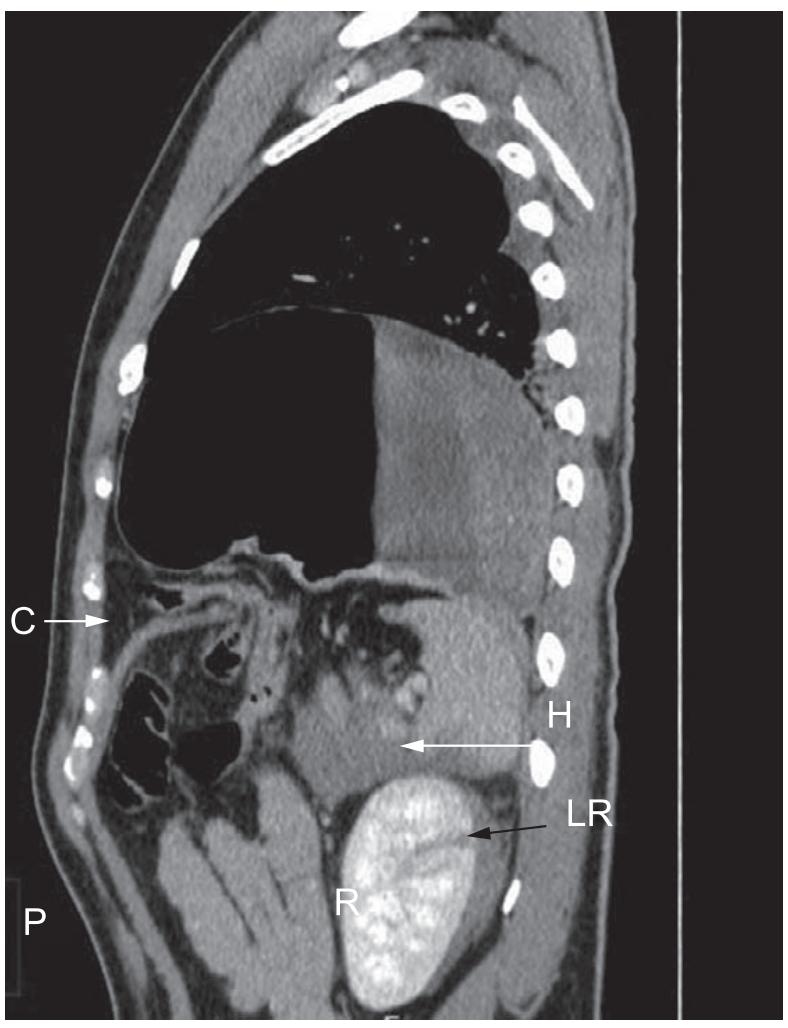

Figura 4 - Imagem MDCT tórax e abdômen, plano sagital mostrando herniação do cólon inferiormente ao estômago (seta C), laceração do rim (seta LR), hematoma anterior ao baço e peri-renal (seta $\mathrm{H}$ ). 
Quando o diafragma contrai, o volume da cavidade torácica aumenta, diminuindo o volume da cavidade abdominal e alterando as pressões intrapleural e intra-abdominal. Durante um ciclo respiratório normal, a pressão intraperitoneal varia de $+2 \mathrm{a}+10 \mathrm{~cm}$ de água e a pressão intrapleural de -5 a $-10 \mathrm{~cm}$ de água o que resulta em um gradiente pleuroperitoneal de $+7 \mathrm{a}+20 \mathrm{~cm}$ de água na posição supina. Com a inspiração máxima ou com a tosse, o diafragma é forçado para baixo e o gradiente da pressão pleuroperitoneal pode aumentar até 100 $\mathrm{cm}$ de água. ${ }^{8,9}$

No trauma, a aplicação de altas pressões pode elevar este gradiente acima de $1000 \mathrm{~cm}$ de água. Este gradiente é transmitido como energia cinética através dos domos diafragmáticos excedendo a complacência do músculo e seus tendões causando roturas radiais ou avulsão do mesmo, principalmente na região póstero-lateral esquerda, embriologicamente um ponto mais fraco ao redor da abertura esofagiana. ${ }^{6,10,11}$ A lesão do diafragma esquerdo ocorre em 68,5 a $87 \%$ das vezes enquanto o diafragma direito é acometido de 13 a $27,3 \%$ e lesões bilaterais, mais raras, ocorrem entre 2 a 4,5\%. ${ }^{6,12}$ Esta diferença de predominância para o lado esquerdo pode estar relacionada à maior vulnerabilidade da região póstero-lateral esquerda como também a proteção que o fígado oferece absorvendo e atenuando a energia transmitida ao diafragma direito. ${ }^{8}$ Em contrapartida estudos de autopsia sugerem igual incidência entre as lacerações diafragmáticas direita e esquerda, mostrando que grande parte dos casos de laceração à direita estão relacionadas a traumas mais intensos que não chegam a ser diagnosticados em vida. ${ }^{13}$

No caso apresentado a rotura diafragmática foi á esquerda por ser a região de maior vulnerabilidade deste músculo como também por ter sido a colisão diretamente sobre a face lateral esquerda do tórax.

Pelo menos $50 \%$ dos pacientes com trauma abdominal fechado e rotura diafragmática também apresentam lesões em outros órgãos, dentre elas: lesões hepáticas $(48 \%)$, hemotórax e ou pneumotórax (47\%), lesão esplênica (35\%), fratura de costela $(23 \%)$, lesão de intestino $(23 \%)$, rins $(16 \%)$, fratura pélvica $(14 \%)$, trauma craniano $(11 \%)$, lesão de aorta $(4 \%)$ e trauma espinhal $(4 \%) .{ }^{8}$ No caso apresentado a lesão concomitante foi do baço que motivou a esplenectomia e o hematoma de retroperitôneo que foi tamponado com gelfoan. A mortalidade da lesão diafragmática unilateral isolada é zero. ${ }^{14,15} \mathrm{~A}$ mortalidade estará relacionada à presença de lesões associadas. ${ }^{8,16}$ Beiji et al. relataram $14,7 \%$ de mortalidade quando a rotura diafragmática se relacionava a lesões tóraco-abdominais e que os pacientes com lesões torácicas são aqueles com maior incidência de óbito. ${ }^{14} A$ rotura bilateral é incomum e está associada a altas taxas de mortalidade ${ }^{2}$ atingindo taxas de $100 \%$ em séries com pequeno número de casos, refletindo o impacto da grande quantidade de energia transmitida e a extensão das lesões viscerais em ambas as cavidades, acompanhadas de instabilidade hemodinâmica. ${ }^{6,12}$

O diagnóstico muitas vezes não é óbvio e passa despercebido na fase aguda do trauma em 7 a $66 \%$ dos pacientes com múltiplas lesões, particularmente nos casos de lesão do diafragma direito. ${ }^{7}$ Por isso a herniação de vísceras abdominais é algumas vezes descrita como uma complicação tardia. Esta herniação tardia e progressiva resulta em dor abdominal e/ou torácica crônica, constipação, estrangulamento ou perfuração de víscera abdominal, dispnéia, infecção respiratória devido à compressão pulmonar no lado afetado. ${ }^{17,18}$ Pacientes com trauma abdominal prévio e sinais de obstrução intestinal devem ser avaliados para a presença de lesão diafragmática não diagnosticada. ${ }^{16}$ Outra complicação descrita é a paralisia do diafragma que apesar de ser mais frequente em lesão cervical pode ocorrer em traumas tóraco-abdominais e no procedimento cirúrgico para o reparo do diafragma. ${ }^{16,19}$

O quadro clínico da rotura diafragmática pode variar desde forma leves com desconforto pleural ou dor abdominal somente, até sinais de sofrimento vascular ou estrangulamento de alças intestinais. ${ }^{6}$ Grimes et al. descreveram três fases clínicas de apresentação da lesão traumática do diafragma. A fase aguda compreende desde a lesão inicial até sua recuperação e caracteriza-se por dor abdominal, presença de outras lesões (parede torácica/abdominal, pelve, cabeça, extremidades, hemopneumotórax, vísceras abdominais), instabilidade hemodinâmica, insuficiência respiratória, diminuição da ventilação pulmonar no lado afetado, ausculta de ruídos intestinais na caixa torácica (patognomônico), alteração radiológica e achado cirúrgico. A fase latente ocorre de horas a semanas após a injúria. Caracteriza-se por queixas gastrointestinais altas, dispnéia e cianose, taquicardia, dor subesternal e dor referida no ombro, agitação, dispnéia exacerbada com o decúbito dorsal horizontal, timpanismo torácico com diminuição da 
ventilação no hemitórax comprometido, ausculta de ruídos hidroaéreos no tórax e anormalidades da radiografia torácica. A fase obstrutiva ocorre meses a anos após o trauma com o estrangulamento da víscera herniada. Os estrangulamentos ocorrem nos primeiros 3 anos, em $85 \%$ dos casos, e se caracterizam por náuseas/vômitos, sintomas de obstrução/isquemia intestinal, dificuldade respiratória crônica secundária a atelectasia, desvio do mediastino, tríade de Borchardt (dor abdominal, distensão abdominal e impossibilidade de passagem de sonda nasogástrica) e ausculta de ruídos intestinais no tórax. ${ }^{20}$ No caso apresentado o paciente não apresentava à admissão sinais e sintomas que pudesssem sugerir a lesão do diafragma e tampouco instabilidade hemodinâmica compatível com extensa lesão visceral. O diagnóstico da lesão diafragmática pode ser feito antes da cirurgia através dos exames de imagem.

No diagnóstico da ruptura do músculo diafragma, além do quadro clínico, dentre os exames mais comumente utilizados, a radiografia do tórax que é mais acessível, pode revelar elevação e/ou irregularidade do contorno do diafragma, presença do estômago ou alças intestinais na cavidade torácica com ou sem constricção da viscera no local da lesão (sinal do colarinho), deslocamento hepático, sonda nasogástrica visível no tórax, deslocamento do mediastino e atelectasias dos lobos inferiores. , 12,21,22 Outras vezes sinais de derrame pleural (hemo/hemopneumotórax) na radiografia do tórax podem representar a presença de vísceras abdominais e/ou trauma torácico. ${ }^{23} \mathrm{~A}$ acurácia do diagnóstico através da radiografia de tórax é quatro vezes maior $(62 \times 17 \%)$ para lesões diafragmáticas à esquerda. ${ }^{22,24}$ Entre 20 e $50 \%$ das radiografias de tórax iniciais de pacientes com rotura diafragmática diagnosticada tardiamente são normais ${ }^{3,22}$ mostrando que este método diagnóstico é insuficiente para excluir lesão diafragmática em paciente com história de mecanismo de trauma que potencialmente favorece esta lesão. ${ }^{6}$

O ultrassom é um método diagnóstico pouco usado, pois a avaliação da continuidade do diafragma não é fácil de ser confirmada. Mihos et al. mostraram acurácia diagnóstica para este método de $26 \%$, porém há estudos que mostram taxa zero de detecção de lesão diafragmática pelo ultrassom. ${ }^{24}$ A tomografia é um método útil neste diagnóstico, utilizada para pacientes estáveis do ponto de vista hemodinâmico e os sinais aceitos e sugestivos de lesão diafragmática incluem descontinuidade do diafragma, herniação de vísceras abdominais com "sinal do colarinho" (constrição de alças e mesentério ao nível da herniação), "sinal da corcova" quando na reformatação coronal há herniação da parte arredondada do fígado gerando imagem de massa em forma de corcova, "Band sign" ou sinal da banda representado por linha radioluscente atravessando a imagem do fígado ao nível da borda diafragmática rota. "Sinal da víscera dependente" quando as vísceras abdominais herniadas se deslocam posteriormente com a gravidade e tocam as costelas. , 21,24 Outros sinais encontrados são: espessamento da crura (pilar diafragmático) em decorrência da retração do musculo diafragma, posicionamento anormal de sonda naso-enteral e lesão contígua como, por exemplo, lesão pulmonar esquerda e laceração esplênica. Os tomógrafos multidetectores (MDCT) de 4 e 16 canais apresentam sensibilidade de 82 a $87 \%$ e especificidade de 72 a $99 \%$ no diagnóstico de hérnia diafragmática, enquanto os aparelhos (MDCT) de 256 canais parecem apresentar resultados ainda melhores devido a capacidade de reconstrução isométrica. ${ }^{23,25}$ Pacientes sob ventilação mecânica podem apresentar resultados falso negativos se a pressão respiratória positiva empurrar as vísceras herniadas de volta para o abdômen. ${ }^{25}$

A Ressonância Nuclear Magnética oferece imagens excelentes de tecidos moles peridiafragmáticos com acurácia no diagnóstico de rotura do diafragma. Porém não é um exame prático para submeter um paciente muitas vezes instável e com entubação oro traqueal, o que limita seu uso na fase aguda. Sua aplicação maior está nos casos de herniação diafragmática crônica. ${ }^{26}$

É também importante pensar em hérnia diafragmática nos pacientes politraumatizados com diagnóstico de derrame pleural de qualquer natureza que necessitem de drenagem torácica, pois durante a drenagem pode haver perfuração iatrogênica de vísceras herniadas, no momento da drenagem, com aumento da morbimortalidade..$^{6,27}$

Em resumo, alto índice de suspeita diagnóstica de rotura diafragmática em trauma fechado acompanhado de investigação radiológica adequada contribui para o diagnóstico correto e precoce. Nos casos submetidos à cirurgia, a avaliação rotineira e meticulosa do diafragma na procura de pequenas lacerações, bem como avaliações seriadas através de exames de imagem nos casos não operados, diminui o risco de complicações tardias. O tratamento cirúrgico na fase aguda resulta em boa evolução na maior parte dos pacientes e previne as complicações. 


\section{REFERÊNCIAS}

1. Rubikas R. Diaphragmatic injurioes. Eur J Cardiothorac Surg. 2001;20:53-7. http://dx.doi.org/10.1016/S10107940(01)00753-9

2. Lewis JD, Starnes SL, Pandalai PK, et al. Traumatic diaphragmatic injury: experience from a level I trauma center. Surgery. 2009;146:578-84. PMid:19789015. http://dx.doi.org/10.1016/j.surg.2009.06.040

3. Davis JW, Eghbalieh B. Injury to the diaphrag. In: Feliciano D, Mattox K, Moore E, editors. Trauma. 6th ed. New York: Mc Graw Hill; 2008. p. 623-35.

4. Drews JA, Mercer C, Benfield JR. Acute diaphragmatic Injuries. Ann Thorac Surg. 1973;16:67-88. http://dx.doi. org/10.1016/S0003-4975(10)65813-4

5. Freeman T, Fischer RP. The inadequacy of peritoneal lavage in diagnosing acute diaphragmatic rupture. J Trauma. 1976;16:538-41. http://dx.doi. org/10.1097/00005373-197607000-00004

6. Morgan BS, Watcyn-Jones T, Garner JP. Traumatic diaphragmatic injury. J R Army Med Corps. 2010;156:139-44. PMid:20919612.

7. Shah R, Sabanathan S, Mearns AJ, ChoudhuryAK. Traumatic rupture of diaphragm. Ann Thorac Surg. 1995;60:1444-9. http://dx.doi.org/10.1016/0003-4975(95)00629-Y

8. Rodriguez-Morales G, Rodriguez A, Shatney $\mathrm{CH}$. Acute rupture of the diaphragm in blunt trauma: analysis of 60 patients. J Trauma 1986; 26:438. http://dx.doi. org/10.1097/00005373-198605000-00005

9. Saladyga AT, Johnson JM, Steinberg SR. Diaphragmatic hernias. New York: Medscape Reference; c2010 [cited 2010 July 12]. Available from: http://emedicine.medscape. com/article/428055

10. Hedblom, CA. Diaphragmatic hernia: a study of three hundred and seventy eight cases in which operation was performed. JAMA. 1925;85:947.

11. Fregnani JHTG, Macéa JR, Barros DB. Cirurgia no hiato esofágico: a identificação correta das estruturas anatômicas. Rev Bras Videocir [Internet]. 2005 [cited 2011 July 25];3:15-20. Available from: http://www.sobracil.org. br/revista/rv030301/rbvc030301_15.pdf. Portuguese.

12. Gwely NN. Outcome of blunt diaphragmatic rupture. Analysis of 44 cases. Asian Cardiovasc Thorac Ann. 2010;18:240-3. PMid:20519291.

13. Scharff JR, Naunheim KS. Traumatic diaphragmatic injuries. Thorac Surg Clin. 2007;17:81-5. PMid:17650700. http://dx.doi.org/10.1016/j.thorsurg.2007.03.006
14. Beigi AA, Masoudpour $H$, Sehhat $S$, Khademi EF. Prognostic factors and outcome of traumatic diaphragmatic rupture. Ulus Travma Acil Cerrahi Derg. 2010;16:215-9. PMid:20517745.

15. Sarna S, Kivioja A. Blunt ruptureof the diaphragm. A retrospective analysis of 41 patients. Ann Chir Gynaecol. 1995;84:261-5. PMid:8702198.

16. Chen JC, Wilson SE. Diaphragmatic injuries: recognition and management in sixty-two patients. Am Surg. 1991;57:810-5. PMid:1746800.

17. Muroni M, Provenza G, Conte S, et al. Diaphragmatic rupture with right colon and small intestine herniation after blunt trauma: a case report. J Med Case Rep. 2010;4:289-91. PMid:20735836. PMCid:2936927. http://dx.doi.org/10.1186/1752-1947-4-289

18. Galimberti A, Casagrande A, Compagnoni BM, et al. Late post-traumatic diaphragmatic hernia: unusual cause of colonic occlusion. Chir Ital. 2001;53:551-4. PMid:11586575

19. Payne JH Jr, Yellin AE. Traumatic diaphragmatic hernia. Arch Surg. 1982;117:18.

20. Grimes O. Traumatic injuries of the diaphragm. Am J Surg. 1974;128:175-81. http://dx.doi.org/10.1016/00029610(74)90090-7

21. Chen HW, Wong YC, Wang LJ, et al. Computed tomography in left-sided and right-sided blunt diaphragmatic rupture: experience with 43 patients. Clin Radiol. 2010;65:206-12. PMid:20152276. http://dx.doi. org/10.1016/j.crad.2009.11.005

22. lochun S, Ludig T, Walter F, et al. Imaging of diaphragmatic injury: a diagnostic challenge. Radiographics. 2002;22:S103-S118. PMid:12376604.

23. Stein DM, York GB, Boswell S, Shanmuganathan K, Haan JM, Scalea TM. Accuracy of computed tomography (CT) scan in the detection of penetrating diaphragm injury. $J$ Trauma. 2007;63:538-43. PMid:18073598. http://dx.doi. org/10.1097/TA.0b013e318068b53c

24. Mihos P, Potaris K, Gakidis J, et al. Traumatic rupture of the diaphragm: experience with 65 patients. Injury. 2003;34:169-72. http://dx.doi.org/10.1016/S0020-1383(02)00369-8

25. Bodanapally UK, Shanmuganathan K, Mirvis SE, et al. MDCT diagnosis of penetrating diaphragm injury. Eur Radiol. 2009;19:1875-81. PMid:19333606. http://dx.doi. org/10.1007/s00330-009-1367-9

26. Eren S, Fahri C. Diaphragmatic hernia: diagnostic approaches with review of the literature. Eur J Radiol. 2005; 54:448-59. PMid:15899350. http://dx.doi.org/10.1016/j. ejrad.2004.09.008

27. Clarke DL, Greatorex B, Oosthuizen GV, Muckart DJ. The spectrum of diaphragmatic injury in a busy metropolitan surgical service. Injury. 2009;40:932-7. PMid:19539925. http://dx.doi.org/10.1016/j.injury.2008.10.042 
Conflito de interesse : Não há.

Submetido em: 28 de Julho de 2011

Aceito em: 31 de Agosto de 2011

Correspondência: Antonio Carlos Nogueira

Rua Dom Armando Lombardi, 701/52

CEP : 05616-011 - São Paulo/SP - Brasil.

E-mail : diaforese@hu.usp.br 
